\title{
The Determinants of Aggregate Wealth
}

\author{
Susheng WANG*
}

January, 1999

\begin{abstract}
This paper analyzes aggregate wealth based on separable risk aversion and intertemporal substitution. In an infinite-period OG model with survival uncertainty, economic growth, and permanent and temporary income shocks, our closed-form solution permits us to derive rich behavioral conclusions and to assess the relative importance of the major components of aggregate wealth. In particular, the separation of the attitudes towards risk and substitution allows us to disentangle behaviors relating to the intertemporal substitution motive from behaviors relating to the precautionary motive.
\end{abstract}

${ }^{*}$ I would like to thank an associate editor for helpful comments that have improved the paper substantially. All errors are mine. 


\section{Introduction}

Recently, the role of precautionary saving against uninsurable income uncertainty has drawn great attention, as several leading researchers have claimed that precautionary saving constitutes at least half of aggregate wealth [see Skinner (1988), Zeldes (1989) and Caballero (1990, 1991)]. However, existing theories on aggregate wealth have been based on expected utilities with either constant absolute risk aversion (CARA) or constant relative risk aversion (CRRA). As we know, these two utilities either do not permit the notion of intertemporal substitution or entangle the latter with risk aversion. As a result, the behaviors relating to intertemporal substitution are mixed with the behaviors relating to risk aversion. As Irvine and Wang (1994) point out, intertemporal substitution is as important as a precautionary motive in determining aggregate wealth. Therefore, the separation of the two factors may be a key to our understanding of aggregate wealth.

We will adopt Weil's (1993) non-expected CARA utility, which has two parameters to represent risk aversion and intertemporal substitution separately. Weil (1993) limits his analysis to individual saving. We will embed Weil's (1993) model in an infiniteperiod, overlapping-generations model with survival uncertainty to extend his work to the analysis of aggregate wealth. In addition, we will allow economic growth with permanent and temporary output shocks. By separating the intertemporal substitution motive from the precautionary motive, and by allowing income growth, we hope to gain a better understanding of aggregate wealth. Note here that the presence of economic growth is important to the behavior of non-precautionary wealth.

Due to the special features of Weil's utility, our aggregate wealth can be divided into a precautionary component and a non-precautionary component. Risk aversion is only relevant to precautionary wealth, while the elasticity of intertemporal substitution is primarily relevant to non-precautionary wealth. The role of interest rates, economic growth and the rate of time preference in aggregate wealth is closely related to the attitude towards intertemporal substitution, while the role of survival uncertainty, income risk and the persistence of shocks is closely related to the attitude towards risk.

The analysis of individual saving is distinctly different from the analysis of aggregate wealth. In an overlapping-generations model, increases in saving by the young generally imply decreases in saving by the old, and vice versa. If a person saves more when she is young, she will dissave more when she is old so as to run her wealth down to zero before she dies. The movement of aggregate wealth is determined by the net effect of the two opposing forces.

The paper is organized as follows. Section 2 presents a model of aggregate wealth and its solution. Section 3 analytically and numerically analyzes behaviors of aggregate wealth, individual wealth and consumption. Finally, Section 4 concludes the paper with a few remarks. All derivations are included in the Appendix. 


\section{The Model}

\subsection{The Intertemporal Utility}

Let us briefly review the utility formulation in Weil (1993). In addition, we will introduce a special feature, survival rate, into the formulation. This feature is crucial for the discussion of aggregate wealth in a model with infinite periods.

Without any uncertainty, a popular utility is

$$
U_{t}=\sum_{i=0}^{\infty} \beta^{i} c_{t+i}^{\rho}
$$

where $0 \leq \rho \leq 1$ and $0 \leq \beta \leq 1$. Let $p$ be the probability of surviving through period $t+1$, given the fact that one is alive in period $t$ :

$$
p \equiv \text { Prob.(survive through period } t+1 \mid \text { alive in period } t \text { ). }
$$

We call $p$ the survival rate. The unconditional probability of reaching age $t$ is $p^{t-1}$. With this survival uncertainty, (2.1) should be revised to ${ }^{1}$

$$
U_{t}=\sum_{i=0}^{\infty}(p \beta)^{i} c_{t+i}^{\rho} .
$$

(2.2) represents the same preferences as the following utility:

$$
U_{t}=\left[(1-p \beta) \sum_{i=0}^{\infty}(p \beta)^{i} c_{t+i}^{\rho}\right]^{1 / \rho}
$$

The latter formulation allows $\rho$ to be negative: $\rho \in(-\infty, 1]$. In particular, the case with $\rho=0$ can be obtained by taking the limit $\rho \rightarrow 0$, i.e.,

$$
U_{t}=(1-p \beta) \sum_{i=0}^{\infty}(p \beta)^{i} \ln c_{t+i} .
$$

The elasticity of intertemporal substitution for the utility in $(2.3)$ is $\eta \equiv \frac{1}{1-\rho}$.

The $U_{t}$ in $(2.3)$ satisfies the following iterative process:

$$
U_{t}=\left[(1-p \beta) c_{t}^{\rho}+p \beta U_{t+1}^{\rho}\right]^{1 / \rho} .
$$

Conversely, under some minor conditions (restrictions on the consumption space ${ }^{2}$ ), any $\left\{U_{t}\right\}_{t=0}^{\infty}$ satisfying (2.4) must be the $U_{t}$ in (2.3). The advantage of (2.4) is that it can be used for the definition of a more general utility.

\footnotetext{
${ }^{1} \mathrm{As}$, for example, in Caballero (1991) and Irvine and Wang (1994).

${ }^{2}$ E.g., Epstein and Zin (1989).
} 
If future consumption is random, then $U_{t+1}$ is random. In this case, let $\hat{U}_{t+1}$ be the certainty equivalent of $U_{t+1}$ and replace (2.4) by

$$
U_{t}=\left[(1-p \beta) c_{t}^{\rho}+p \beta \hat{U}_{t+1}^{\rho}\right]^{1 / \rho}
$$

Here, $\eta \equiv \frac{1}{1-\rho}$ is now the elasticity of intertemporal substitution between current consumption and the certainty equivalent of future consumption. In Weil's (1993) formulation, the certainty equivalent is defined by constant absolute risk aversion $\theta:{ }^{3}$

$$
e^{-\theta \hat{U}_{t+1}}=E_{t}\left(e^{-\theta U_{t+1}}\right)
$$

Here, $\theta=0$ corresponds to risk neutrality, i.e., $\hat{U}_{t+1}=E_{t} U_{t+1}$. Combining (2.5) and (2.6) gives a recursive intertemporal utility formulation:

$$
U_{t}=\left[(1-p \beta) c_{t}^{\rho}+p \beta\left(-\frac{1}{\theta} \ln E_{t} e^{-\theta U_{t+1}}\right)^{\rho}\right]^{1 / \rho}
$$

This formulation has also been axiomatically rationalized by Kreps and Porteus (1978).

\subsection{The Individual Problem}

For each individual, we use Weil's (1993) formulation. For the aggregate economy, we use an overlapping-generations model with a constant population size, infinitely lived individuals with survival risk, and economic growth with permanent and temporary shocks. Instead of dealing with individual consumption demand as in Weil (1993), we concentrate on aggregate wealth in equilibrium.

The individuals in our model are the same when they are born, with the same preferences as represented by the intertemporal utility in (2.7), and the same endowment as defined by an initial nonhuman wealth $A_{0}$ and a lifetime income process $\left\{y_{t}\right\}_{t=0}^{\infty}$. There is also a capital market for lending and borrowing at a constant interest rate $r .{ }^{4}$ We denote $\hat{r} \equiv 1+r$.

Given survival rate $p$ and population size 1 for the whole society in any period, the population size of individuals dying in any period is $1-p$. The population size of the newborns in each period is assumed to be $1-p$, which maintains the population size at 1 .

\footnotetext{
${ }^{3}$ In Epstein and Zin's (1989) formulation, the certainty equivalent is defined by constant relative risk aversion $\theta$ :

$$
\hat{U}_{t+1}^{1-\theta}=E_{t}\left(U_{t+1}^{1-\theta}\right) .
$$

This formulation, however, does not permit a closed-form solution for our problem.

${ }^{4}$ The interest rate $r$ here can be considered as the real interest rate for an annuity or a long-term indexed bond.
} 
Saving and economic growth should naturally be closely related to each other. Economic growth may thus be important to aggregate wealth. To incorporate it into our model, we propose an income process of the form:

$$
y_{t+1}=(\hat{a}-\lambda) \hat{a}^{t} y_{0}+\lambda y_{t}+\varepsilon_{t+1},
$$

where $a \in \mathbb{R}, \hat{a} \equiv 1+a, \lambda \in[0,1]$, and $\left\{\varepsilon_{t}\right\}$ is white noise. Let $\bar{y}_{t} \equiv E\left(y_{t}\right)$. In the steady state where $y_{t}=\bar{y}_{t}$ for all $t,(2.8)$ implies $y_{t}=\hat{a}^{t} y_{0} \cdot{ }^{5}$ That is, output grows at a constant rate $a$ in the steady state. The persistence of income shocks is measured by $\lambda$, with $\lambda=0$ corresponding to a purely transitory shock, and $\lambda=1$ corresponding to a permanent shock. That is, in our formulation, income has three components: the growth component $x_{t} \equiv(\hat{a}-\lambda) \hat{a}^{t} y_{0}$, the persistent component $\lambda y_{t}$, and the random component $\varepsilon_{t+1}$. Each component has a unique role in aggregate wealth.

Each individual faces the following lifetime utility maximization problem:

$$
\begin{aligned}
V\left(A_{t-1}, x_{t-1}, y_{t}\right)=\max & {\left[(1-p \beta) c_{t}^{\frac{\eta-1}{\eta}}+p \beta\left(-\frac{1}{\theta} \ln E_{t} e^{-\theta V\left(A_{t}, x_{t}, y_{t+1}\right)}\right)^{\frac{\eta-1}{\eta}}\right]^{\frac{\eta}{\eta-1}} } \\
\text { s.t. } & A_{t}=\hat{r} A_{t-1}+y_{t}-c_{t} \\
& y_{t+1}=x_{t}+\lambda y_{t}+\varepsilon_{t+1} \\
& \text { given } A_{0}, y_{0},
\end{aligned}
$$

where $E_{t}$ is the expectations operator conditional on information $\Phi_{t}$ available at time $t, \quad c_{t}$ is consumption, $A_{t}$ is wealth, and $\beta$ is the discount factor of time preference. Let

$$
\delta \equiv(p \beta \hat{r})^{\eta}, \quad \varepsilon^{*} \equiv \frac{1}{\varphi} \ln E_{t} e^{-\varphi \varepsilon_{t+1}}, \quad \varphi \equiv \frac{\theta}{\hat{r}-\lambda}\left(\hat{r}-\delta^{1 / \eta}\right)^{\frac{\eta}{\eta-1}}(\hat{r}-\delta)^{\frac{1}{1-\eta}} .
$$

The Appendix derives the solution in a feedback form depending on the state $\left(A_{t-1}, x_{t-1}, y_{t}\right):{ }^{6}$

$$
c_{t}=(\hat{r}-\delta)\left[A_{t-1}+\frac{x_{t}+r y_{t}-\varepsilon^{*}}{r(\hat{r}-\lambda)}\right] .
$$

By (2.10) and the budget conditions in (2.9), we can express $c_{t}$ in a recursive form:

$$
c_{t+1}=\delta c_{t}+\frac{\hat{r}-\delta}{\hat{r}-\lambda}\left(\frac{a}{r} x_{t}+\varepsilon_{t+1}+\varepsilon^{*}\right),
$$

${ }^{5}$ We can also consider a more general form:

$$
y_{t+1}=(\hat{a}-\lambda) \hat{a}^{t} \hat{y}+\lambda y_{t}+\varepsilon_{t+1} .
$$

Then, in the steady state, $y_{t}=\hat{a}^{t} \hat{y}+\lambda^{t}\left(y_{0}-\hat{y}\right)$, which has a steady growth rate $a$ and a diminishing deviation term $\lambda^{t}\left(y_{0}-\hat{y}\right)$. When there is no growth, the general form becomes Weil's (1993) formula of income formulation.

\footnotetext{
${ }^{6}$ The difference between our income process and Weil's (1993) forces us to solve the consumption demand independently. When the growth rate is zero, our solution is the same as Weil's (1993) solution (with $\hat{y}=y_{0}$ ).
} 
which indicates the economic role of $\delta$. By (2.10) and the budget equations in (2.9) again, we can also express $A_{t}$ in a recursive form:

$$
A_{t}=\delta A_{t-1}+\frac{\delta-\lambda}{\hat{r}-\lambda} y_{t}-\frac{\hat{r}-\delta}{r(\hat{r}-\lambda)}\left(x_{t}-\varepsilon^{*}\right),
$$

which can then be used recursively to yield

$$
A_{t}=\delta^{t} A_{0}+\frac{\delta-\lambda}{\hat{r}-\lambda} \sum_{i=1}^{t} \delta^{t-i} y_{i}-\frac{\hat{r}-\delta}{r(\hat{r}-\lambda)} \sum_{i=1}^{t} \delta^{t-i} x_{i}+\frac{\hat{r}-\delta}{r(\hat{r}-\lambda)} \frac{1-\delta^{t}}{1-\delta} \varepsilon^{*}
$$

\subsection{The Aggregate Equilibrium}

As the population size of the age $t$ group is $(1-p) p^{t-1}$, aggregate wealth is

$$
W=(1-p) \sum_{t=1}^{\infty} p^{t-1} A_{t}
$$

At any moment in time, there are $1-p$ newborns, demanding a total wealth endowment $(1-p) A_{0}$. At the same time, $1-p$ individuals die, leaving a total accidental wealth $(1-p) W$. Following Caballero (1991), the equilibrium condition is to equalize the total supply of wealth and the total demand for wealth, which implies

$$
A_{0}=W
$$

Substituting (2.13) into (2.11) and then into (2.12) gives an equation for $W$, which can then be solved for the equilibrium aggregate wealth:

$W^{*}=\frac{1-p \delta}{1-\delta} \frac{1-p}{p} \frac{\delta-\lambda}{\hat{r}-\lambda} \sum_{t=1}^{\infty} \sum_{i=1}^{t} p^{t} \delta^{t-i} y_{i}-\frac{\hat{a}(1-p)(\hat{a}-\lambda)(\hat{r}-\delta) y_{0}}{r(\hat{r}-\lambda)(1-\delta)(1-\hat{a} p)}+\frac{(\hat{r}-\delta) \varepsilon^{*}}{r(\hat{r}-\lambda)(1-\delta)}$

The existence of this equilibrium solution needs the following assumption. ${ }^{7}$

Assumption 1. $p \delta<1$ and $p \hat{a}<1$.

It is not surprising that the two conditions in Assumption 1 guarantee the existence of a stationary equilibrium - the two conditions are statements about the curvature of an agent's felicity function and the ways in which the turnover of the population is sufficiently rapid compared to the economy's income growth trend. These type of conditions are fairly common in growth models with linear production technologies.

\footnotetext{
${ }^{7}$ For the positivity of consumption, we need to impose additional conditions on the parameters. For our purposes, there is, however, no need to spell out the conditions. For realistic parameter values, as chosen in Section 3, consumption is positive for all age groups.
} 


\subsection{The Steady State Equilibrium}

Notice that the incomes in (2.14) are realized incomes. If the sample of incomes is in the steady state where $y_{t}=\bar{y}_{t}$ for all $t$, then

$$
y_{t}=y_{0} \hat{a}^{t}
$$

and

$$
W^{*}=\frac{\hat{a}(1-p)[r(\delta-\lambda)-(\hat{r}-\delta)(\hat{a}-\lambda)]}{r(1-\delta)(\hat{r}-\lambda)(1-\hat{a} p)} y_{0}+\frac{\hat{r}-\delta}{r(\hat{r}-\lambda)(1-\delta)} \varepsilon^{*} .
$$

If $\varepsilon_{t+1}$ is conditionally normally distributed: $\varepsilon_{t+1} \mid \Phi_{t} \sim N\left(0, \sigma^{2}\right)$, then

$$
\varepsilon^{*}=\frac{\theta \sigma^{2}}{2(\hat{r}-\lambda)}\left(\hat{r}-\delta^{1 / \eta}\right)^{\frac{\eta}{\eta-1}}(\hat{r}-\delta)^{\frac{1}{1-\eta}}
$$

Substituting (2.13) and (2.16) into (2.11) then gives

$$
A_{t}^{*}=\frac{r(\delta-\lambda)-(\hat{r}-\delta)(\hat{a}-\lambda)}{r(\hat{r}-\lambda)(\hat{a}-\delta)}\left[1+\frac{a(1-\delta p)}{(1-\delta)(1-\hat{a} p)}\left(\frac{\delta}{\hat{a}}\right)^{t}\right] \hat{a} y_{t}+\frac{(\hat{r}-\delta) \varepsilon^{*}}{r(\hat{r}-\lambda)(1-\delta)} .
$$

Further substituting (2.17) into (2.10) gives

$$
c_{t}^{*}=\frac{\hat{r}-\delta}{\hat{r}-\lambda}\left\{\left[\hat{a}-\lambda+\frac{r(\delta-\lambda)-(\hat{r}-\delta)(\hat{a}-\lambda)}{(1-\delta)(1-\hat{a} p)}(1-\delta p)\left(\frac{\delta}{\hat{a}}\right)^{t-1}\right] \frac{a y_{t}}{r(\hat{a}-\delta)}+\frac{\varepsilon^{*}}{1-\delta}\right\} .
$$

Several remarks are in order. First, even though adjustments in wealth can be passed to future generations in this model, there is no voluntary bequest motive. Consequently, inheritance comes solely from involuntary bequests.

Second, the absence of income insurance in our model is realistic and standard in these models, due to the moral hazard problems that would be associated with its introduction. In other words, we deal with uninsurable income risks.

Third, the absence of an insurance market for lifetime uncertainty in our model is based on Yaari's (1964) result that the conditions governing the evolution of the equations of motion for saving and consumption are the same in the no-lifetime uncertainty case as in the case where lifetime is uncertain but with an annuity market where the rate of interest is 'fair'. Thus, if the terminal conditions of the problem were the same in each of these cases, the actual path of wealth accumulation would be the same, and the inclusion of a market for life insurance would be equivalent to the path under a certain lifetime. $^{8}$

\footnotetext{
${ }^{8}$ Kotlikoff (1988) has, however, argued that 'fair' annuities are difficult to supply in practice because of agent heterogeneity and self-selection.
} 
Finally, since our model does not include physical capital, we are unable to discuss many issues in growth theory. However, we think that physical capital plays a crucial role only in the transition state of an economy. Since our interest is the aggregate wealth in a steady state, it should not make a major difference in our conclusions if physical capital were included. Besides that, the tractability of a model without physical capital allows us to analyze some important issues in the literature of wealth accumulation that a growth model cannot easily handle, such as the separation of precautionary and non-precautionary wealth. These are the main two reasons that researchers in the area of aggregate wealth, such as Modigliani and Brumberg (1954), Skinner (1988), Zeldes (1989) and Caballero (1990, 1991), have used exogenous output processes. Here, the constant output growth rate in our model can be treated as the steady-state output growth rate, which should be mainly determined by exogenous factors such as technology and individual preferences.

\section{Analysis}

We now analyze the steady state equilibrium. In our discussions, we restrict $r, \delta, a \in[0,1]$. Also, Assumption 1 will be satisfied in our benchmark.

\subsection{Aggregate Wealth}

Aggregate wealth in (2.16) can be divided into a non-precautionary component $W_{N}^{*}$ and a precautionary component $W_{P}^{*}$ :

$$
\begin{aligned}
& W_{N}^{*} \equiv \frac{\hat{a}(1-p)[r(\delta-\lambda)-(\hat{r}-\delta)(\hat{a}-\lambda)]}{r(1-\delta)(\hat{r}-\lambda)(1-\hat{a} p)} y_{0}, \\
& W_{P}^{*} \equiv \frac{(\hat{r}-\delta) \varepsilon^{*}}{r(\hat{r}-\lambda)(1-\delta)}=\frac{\theta \sigma^{2}\left(\hat{r}-\delta^{1 / \eta}\right)^{\frac{\eta}{\eta-1}}(\hat{r}-\delta)^{\frac{2-\eta}{1-\eta}}}{2 r(\hat{r}-\lambda)^{2}(1-\delta)} .
\end{aligned}
$$

While $W_{N}^{*}$ is unrelated to income uncertainty, $W_{P}^{*}$ results from income uncertainty. While intertemporal substitution has a role in both precautionary and non-precautionary wealth, risk aversion is only relevant to precautionary wealth, and economic growth is only relevant to non-precautionary wealth. This separation would be impossible with a traditional expected utility, for which risk aversion and elasticity of intertemporal substitution are entangled and are represented by a single parameter.

Higher income risk raises aggregate wealth by raising precautionary wealth:

$$
\frac{\partial W^{*}}{\partial \sigma}=\frac{\partial W_{P}^{*}}{\partial \sigma}=\frac{2(\hat{r}-\delta) \varepsilon^{*}}{r(\hat{r}-\lambda)(1-\delta) \sigma}>0 .
$$

This effect has attracted considerable attention recently, ${ }^{9}$ as people generally find that

\footnotetext{
(1994).

${ }^{9}$ E.g., Skinner (1988), Zeldes (1989), Caballero (1990, 1991), Carroll (1992), and Irvine and Wang
} 
income uncertainty is of major importance in aggregate wealth.

As expected, higher risk aversion also raises aggregate wealth by raising precautionary wealth:

$$
\frac{\partial W^{*}}{\partial \theta}=\frac{\partial W_{P}^{*}}{\partial \theta}=\frac{(\hat{r}-\delta) \varepsilon^{*}}{r(\hat{r}-\lambda)(1-\delta) \theta}>0 .
$$

It is interesting to note that attitude towards risk, as measured by $\theta$, has nothing to do with non-precautionary wealth. However, expected utilities, which mix attitude towards risk and attitude towards intertemporal substitution, inevitably entangle precautionary and non-precautionary wealth. The result in (3.3) will thus not be as clear-cut under expected utility.

Precautionary wealth is independent of economic growth, and, if $\delta \leq \lambda$,

$$
\frac{\partial W^{*}}{\partial a}=\frac{\partial W_{N}^{*}}{\partial a}<0
$$

This results from the so-called consumption smoothing motive. ${ }^{10}$

Aggregate income in the steady state is

$$
Y \equiv(1-p) \sum_{t=1}^{\infty} p^{t-1} y_{t}=\frac{\hat{a}(1-p) y_{0}}{1-\hat{a} p}
$$

If we use this expression to replace $y_{0}$ by $Y$, then (2.16) becomes

$$
\frac{W^{*}}{Y}=\frac{r(\delta-\lambda)-(\hat{r}-\delta)(\hat{a}-\lambda)}{r(1-\delta)(\hat{r}-\lambda)}+\frac{\left(\hat{r}-\delta^{1 / \eta}\right)^{\frac{\eta}{\eta-1}}(\hat{r}-\delta)^{\frac{2-\eta}{1-\eta}}}{2 r(\hat{r}-\lambda)^{2}(1-\delta)}(\theta Y)\left(\frac{\sigma}{Y}\right)^{2} .
$$

This formula can be conveniently used for numerical analysis, as we need not assign a value to $Y$. The benchmark parameter values are chosen as

$$
r=5 \%, \quad a=2 \%, \lambda=98 \%, \quad \sigma / Y=20 \%, \quad \theta Y=3, \quad \eta=1.8, \quad p=96 \%, \quad \beta=98 \% \text {. }
$$

All parameter values, except $\eta$, are chosen based on stylized facts, representing the annual data for the United States. The elasticity $\eta=1.8$ is chosen so that $W^{*} / Y$ is close to 3 , which is also a stylized fact. Since the existing literature provides little indication of the range of $\eta$, we borrow the well known stylized fact on $W^{*} / Y$ in the determination of $\eta$. The interest rate $r=5 \%$ and the output growth rate $a=2 \%$ are used widely in the literature [see, for example, Caballero (1990) and Irvine and Wang (1994) for the value of the interest rate]. The discount factor $\beta=98 \%$ means that the rate of time preference is about $2 \%$, which is used in Irvine and Wang (1994). The rate of income uncertainty $\sigma / Y=20 \%$ comes from Caballero (1991), who concludes that $\sigma / Y>10 \%$. A $96 \%$ survival rate implies $16 \%$ population size of retirees (age 65 or older), which is realistic. ${ }^{11}$ The variable $\theta Y$ corresponds to the relative risk

\footnotetext{
${ }^{10}$ Four figures in Carroll and Weil (1993) suggest a long-term negative relation between saving rate and economic growth for Japan, South Korea, Singapore, and Hong Kong.

${ }^{11}$ Here $t$ is the number of working years and the actual age is $t+20$.
} 
aversion, which has often been assigned a value between 2 and 4 [see, for example, Skinner (1988), Zeldes (1989) and Caballero (1990, 1991)]. ${ }^{12}$ Empirical studies suggest a $\lambda$ value that is fairly close to 1 ; we choose $\lambda=0.98$ [see, for example, Prescott (1986) and McCallum (1989, p.194)].

We have

$$
\frac{\partial W_{P}^{*}}{\partial \lambda}=\frac{(\hat{r}-\delta) \varepsilon^{*}}{r(\hat{r}-\lambda)^{2}(1-\delta)}>0, \quad \frac{\partial W_{N}^{*}}{\partial \lambda}=-\frac{a \hat{a}(1-p)(\hat{r}-\delta) y_{0}}{r(1-\delta)(1-\hat{a} p)(\hat{r}-\lambda)^{2}}<0 .
$$

Since higher persistence allows shocks to last longer and thus increases 'intertemporal uncertainty', it boosts precautionary wealth, just as the variability of shocks does. Interestingly, higher persistence also means a more intertemporally stable expected income. With higher persistence, expected future income is more closely related to current income. This works against wealth accumulation. That is, people accumulate less non-precautionary wealth if the expected component of their income is more intertemporally stable. The overall effect of persistence on aggregate wealth is generally positive. In the benchmark (3.4), aggregate wealth increases in $\lambda$ iff $\lambda>80 \%$. Note that if there were no survival risk, the persistence of shocks would not affect non-precautionary wealth. ${ }^{13}$

In the benchmark, we have

$$
\frac{\partial W_{N}^{*}}{\partial \eta}>0, \quad \frac{\partial W_{P}^{*}}{\partial \eta}<0, \quad \frac{\partial W^{*}}{\partial \eta}<0 .
$$

The concept of risk aversion deals with random fluctuations within a period, while the concept of intertemporal substitution deals with expected fluctuations across periods. Stronger preference towards intertemporal substitution means that the individuals are more tolerant towards expected fluctuations in consumption over their lifetime, while stronger risk aversion means that the individuals are less tolerant towards random fluctuations in consumption within a period.

In a growing economy, non-precautionary motives are encouraged by higher tolerance towards expected consumption fluctuations; as a result, non-precautionary wealth increases. ${ }^{14}$ As non-precautionary saving becomes relatively more important, some

\footnotetext{
${ }^{12}$ Since existing empirical estimates of the elasticity of intertemporal substitution are done in models that mix risk aversion with intertemporal substitution, we have to re-estimate the elasticity for our model and we have done so using a stylized fact. However, we have accepted the empirical estimate of risk aversion made in models with conventional expected utility functions, since the risk aversion is often estimated from one-period models for which intertemporal substitution is absent. In other words, while existing estimates of the elasticity of intertemporal substitution are unreliable, the existing estimates of the risk aversion should be reliable.

${ }^{13}$ The intertemporal stability here is a different notion of intertemporal stability from one involving transition paths from one steady state to another. Our economy is an endowment economy at a steady state, and our notion of intertemporal stability applies to the economy at a steady state, rather than to a transition path from a steady state to another.

${ }^{14}$ If there were no economic growth, then consumption would be constant, rather than growing, and our argument and the results in (3.5) would no longer hold.
} 
saving switches from precautionary saving to non-precautionary saving on the margin, implying a smaller precautionary wealth.

The effect of the interest rate on wealth accumulation is always of interest. In the benchmark, we have

$$
\frac{\partial W_{N}^{*}}{\partial r}<0, \quad \frac{\partial W_{P}^{*}}{\partial r}>0, \quad \frac{\partial W^{*}}{\partial r}>0 .
$$

In our OG model with a survival rate, the direction of saving's movement by the old are often not the same as the movement of aggregate wealth. A higher interest rate may induce more non-precautionary saving and result in a higher individual wealth late in one's life [the substitution effect, see the empirical findings in Summers (1981)]. But the individuals die out over time, which means more wealth endowment to newborns. Newborns with a higher wealth endowment may save less (the wealth effect). The net effect of the intergenerational wealth and substitution effects on non-precautionary wealth is negative. Similarly, a higher interest rate may induce less precautionary saving [see the empirical findings in Carroll (1992)] that results in less individual wealth late in one's life. This means less endowment to the newborns, which induces more saving from younger generations. The net effect on precautionary wealth is positive.

In the benchmark, we have

$$
\frac{\partial W_{N}^{*}}{\partial p}<0, \quad \frac{\partial W_{P}^{*}}{\partial p}>0, \quad \frac{\partial W^{*}}{\partial p}<0 .
$$

A higher survival rate means fewer bequests and thus lower non-precautionary wealth. As each individual lives longer, the same degree of income risk in each period will have more effect on one's lifetime consumption plans. Thus, a higher survival rate boosts precautionary wealth.

The discount factor $\beta$ enters the utility in the same way as $p$ does, and their effects on the two wealth components are the same. But their net effect on aggregate wealth is the opposite:

$$
\frac{\partial W_{N}^{*}}{\partial \beta}<0, \quad \frac{\partial W_{P}^{*}}{\partial \beta}>0, \quad \frac{\partial W^{*}}{\partial \beta}>0 .
$$

As each individual increases her preference towards future consumption, more wealth is accumulated against the same degree of income risk in order to protect future consumption. Thus, a higher discount factor boosts precautionary wealth.

More patient individuals may save more (the substitution effect) and have more wealth late in their life. But these individuals die out over time, which means more wealth endowment to newborns. Newborns with a higher wealth endowment may save less (the wealth effect). The net effect of the intergenerational wealth and substitution effects on non-precautionary wealth is negative. Note that, when there is no economic growth, the discount factor has no impact on non-precautionary wealth. 
An interesting issue is the relative importance of economic growth, interest rate, and income uncertainty in aggregate wealth. We will use the elasticities of aggregate wealth as a measure of their importance. Using (2.16), in the benchmark, we have ${ }^{15}$

$$
\begin{array}{rlrl}
e_{r} & \equiv \frac{r}{W^{*}} \frac{\partial W^{*}}{\partial r}=18.7, & \frac{\partial e_{r}}{\partial \eta}>0, & \frac{\partial e_{r}}{\partial \theta}<0 ; \\
e_{\sigma} \equiv \frac{\sigma / Y}{W^{*}} \frac{\partial W^{*}}{\partial(\sigma / Y)}=27.2, & \frac{\partial e_{\sigma}}{\partial \eta}>0, & \frac{\partial e_{\sigma}}{\partial \theta}<0 ; \\
e_{a} \equiv \frac{a}{W^{*}} \frac{\partial W^{*}}{\partial a}=-18.0, & \frac{\partial e_{a}}{\partial \eta}<0, & \frac{\partial e_{a}}{\partial \theta}>0 .
\end{array}
$$

Many recent theoretical and empirical findings suggest that income uncertainty is about twice as important as interest rates. ${ }^{16}$ Our calculated $e_{r}$ and $e_{\sigma}$ confirm this observation. It is, however, surprising to find that economic growth is as important as interest rate.

Regarding the second column in (3.8), a higher elasticity of intertemporal substitution means higher tolerance towards intertemporal fluctuations in consumption, which naturally increases the importance of those factors that favor saving, such as interest rates and income uncertainty. Likewise, it decreases the importance of those factors that favor consumption smoothing, such as economic growth. More tolerant agents react more to interest rate and income uncertainty. Likewise, since $e_{a}$ measures the importance of the consumption smoothing motive, higher tolerance of consumption fluctuations reduces it.

Regarding the third column in (3.8), risk aversion measures intolerance towards consumption fluctuations, which pushes the importance in the other direction, resulting in the opposite signs in the second and third columns. ${ }^{17}$

\subsection{Individual Wealth}

Individual wealth can also be divided into a non-precautionary component and a precautionary component. It is interesting to note that precautionary wealth is independent of age. The reason is as follows. Our model is stationary - tomorrow's shock is like today's shock. The uncertain lifetime framework prevents an agent from anticipating his or her demise for sure, so the agent always behaves as if there is another tomorrow. Death is always a surprise. Hence, an individual's age should not influence his or her decision making on precautionary saving.

\footnotetext{
${ }^{15}$ Caution should be taken here. The elasticity values are marginal values. This means that for, say, a $5 \%$ increase in $\sigma$, we should not conclude that the aggregate wealth will increase by $5 \times 27.2=$ $136 \%$. When $\sigma$ increases from 20 to 21 , with other parameter values being the same, the elasticity drops to 12.5 !

${ }^{16}$ E.g., Modigliani (1954), Skinner (1988), Caballero (1990, 1991), Carroll (1993), and Irvine and Wang (1994).

${ }^{17}$ Are our conclusions in this section robust? For that, we try a few other combinations of parameter values. We find the same conclusions. We are thus confident that our conclusions are robust.
} 
The differences between the equilibrium individual wealth in (2.17) and the individual wealth demand in (2.11) are reflected by the difference in the wealth endowment. Because of the discount factor $\delta$ that applies to the wealth endowment in the wealth demand, the wealth demand by the old behaves similarly to the equilibrium wealth, while the wealth demand by the young behaves differently. Here, we distinguish the young and the old by the average age in the population.

In the benchmark, the individual wealth profile $A_{t}^{*}$ is plotted in the following graph. It has a stylized hump, achieving the maximum at $t=26$ or actual age 46 . The individual finishes off her wealth at $t=60$ or actual age $80 .{ }^{18}$

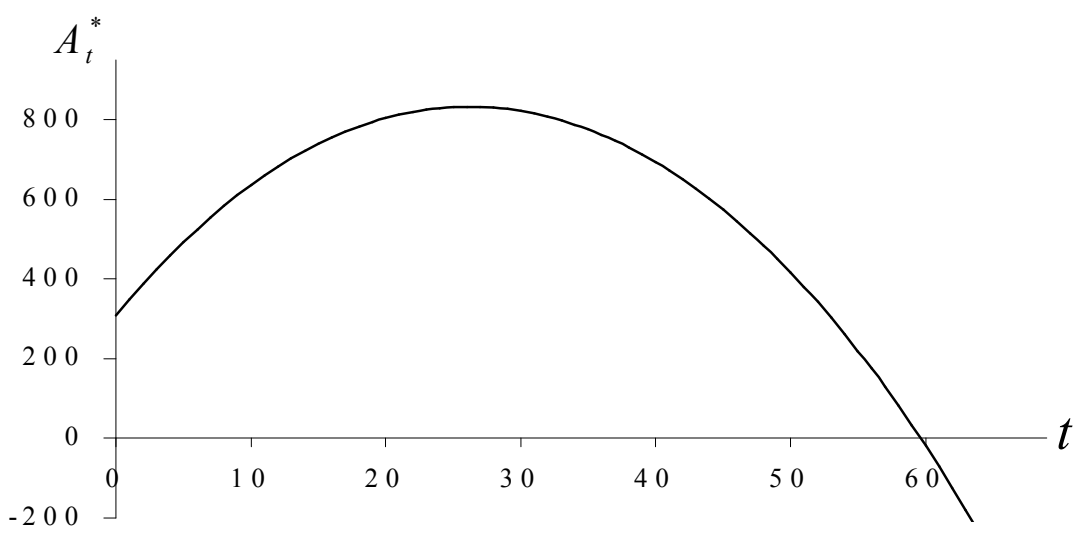

Figure 1. Individual Wealth Profile

\subsection{Consumption}

Weil (1993) has provided a detailed discussion of consumption demand. Here, we discuss only the equilibrium consumption and its comparison with the consumption demand in Weil (1993).

The equilibrium consumption in (2.18) is of the Keynesian type, i.e., a linear function of current income, while the consumption demand in (2.10) obeys the permanent income hypothesis. Without risk, the equilibrium consumption also obeys the permanent income hypothesis.

Let $\mu$ be the marginal propensity to consume out of current income from the consumption demand, and $\mu^{*}$ be the marginal propensity to consume out of current income from the equilibrium consumption. As Weil (1993) points out, $\mu$ is increasing in $\lambda$. Hence, the consumption demand reacts more to permanent than to transitory shocks in income. This is consistent with the well-known prediction by Milton Friedman.

\footnotetext{
${ }^{18}$ Individuals are allowed to have negative wealth. Since they receive an income $y_{t}$ every period, they can afford to borrow to certain extent. The question is whether the transversality condition should be used, which will put a lower bound on wealth. This turns out to be unnecessary in our model. Since the population size of a cohort quickly goes to zero as it ages, our conclusions on aggregate wealth will be the same whether or not we put a lower bound on wealth. The lower bound will affect only a negligible segment of the population.
} 
However, this conclusion is based on a partial equilibrium. By inspecting (2.18), this conclusion is reversed for $\mu^{*}$ if $a \leq r$. Everyone may demand more consumption, but in equilibrium, the relative price will adjust in favor of saving; in our particular case, as fueled by intergenerational wealth transfers in equilibrium, when the interest rate is high, such a price effect is strong enough to reverse the direction.

Although $\mu$ does not depend on age, $\mu^{*}$ increases with age. This is expected because, as one gets older, a smaller proportion of income needs to be saved.

An individual who has stronger preference towards intertemporal substitution, i.e., a larger $\eta$, will have a larger $\mu$. In the benchmark, such a conclusion also holds for $\mu^{*}$. As we know, the elasticity of intertemporal substitution is a measure of tolerance towards intertemporal variation in consumption. With the possibility of early death, there is a chance for individual wealth not to be consumed. Because of that, individuals with stronger preference towards intertemporal substitution choose to consume more when young.

Although $\mu$ is not affected by economic growth, in the benchmark, higher economic growth leads to a lower $\mu^{*}$. Since the equilibrium precautionary saving is not affected by economic growth, higher economic growth reduces the saving rate. See Carroll and Weil (1993) for some empirical findings, as mentioned in Footnote 10. This is due to the so-called consumption smoothing motive.

Weil (1993) shows that an increase in the interest rate increases, as under certainty, the propensity to consume from wealth if the elasticity of intertemporal substitution is greater than one. What will happen in equilibrium where intergenerational transfer of wealth is an important factor? In our benchmark, we have

$$
\frac{\partial \mu^{*}}{\partial r}<0, \quad \frac{\partial c_{t}^{*}}{\partial r}>0
$$

for all $t$. A higher interest rate means a 'higher price' for current consumption (the substitution effect), which encourages non-precautionary saving and results in a lower propensity. The precautionary saving, however, works against such a tendency: a higher interest rate implies a bigger 'wealth' (the wealth effect), which enhances the effects of income uncertainty and lowers the precautionary saving. The precautionary motive dominates, driven by the wealth effect and fueled by intergenerational wealth transfers.

Finally, in the benchmark, we have

$$
\frac{\partial \mu^{*}}{\partial p}<0, \quad \frac{\partial c_{t}^{*}}{\partial p}<0
$$

for all $t$. A reduction in the survival uncertainty suggests a longer life expectancy, which naturally reduces the consumption propensity and encourages saving. Notice here that aggregate wealth is not increased by an increase in saving from this source, see (3.7). Intergenerational wealth transfers and the distribution of the population are the key underlying factors that reverse the direction of aggregate wealth. 


\section{Concluding Remarks}

Using Weil's (1993) utility that separates intertemporal substitution from risk aversion, we are in a better position to understand the roles of interest rates, income risk, economic growth, survival uncertainty, the rate of time preference and the persistence of shocks in aggregate wealth. The division of precautionary and non-precautionary wealth makes it possible to analyze how intertemporal substitution and risk aversion affect wealth accumulation. Risk aversion is only relevant to precautionary wealth, while intertemporal substitution is more relevant to non-precautionary wealth.

The importance of the two most important factors in aggregate wealth — interest rates and income risk - is determined separately by intertemporal substitution and risk aversion. More restrictive expected utilities that mix intertemporal substitution with risk aversion cannot properly disentangle the role of interest rates from the role of income risk in aggregate wealth. ${ }^{19}$

As our analysis indicates, individual saving and aggregate wealth are distinctly different matters. One is a demand concept, while the other involves an equilibrium process. The saving's movement in some age groups, especially the old, can be the opposite of the movement of aggregate wealth. Even consumption demand and equilibrium consumption can move in opposite directions.

\section{Appendix}

This appendix solves problem (2.9) and derives the formulas in Section 2.

\section{A.1. Solving the Consumer Problem}

Problem (2.9) is

$V^{\frac{\eta-1}{\eta}}\left(A_{t-1}, x_{t-1}, y_{t}\right)=$ optimize $(1-p \beta) c_{t}^{\frac{\eta-1}{\eta}}+p \beta\left[-\frac{1}{\theta} \ln E_{t} e^{-\theta V\left(\hat{r} A_{t-1}+y_{t}-c_{t}, x_{t}, y_{t+1}\right)}\right]^{\frac{\eta-1}{\eta}}$.

Assume

$$
V\left(A_{t-1}, x_{t-1}, y_{t}\right) \equiv \phi_{1} A_{t-1}+\phi_{2} y_{t}+\phi_{3} x_{t-1}+\phi_{4},
$$

with constants $\phi_{i}$. With (A.2), the right side of (A.1) becomes

$(1-p \beta) c_{t}^{\frac{\eta-1}{\eta}}+p \beta\left[\phi_{1}\left(\hat{r} A_{t-1}+y_{t}-c_{t}\right)+\phi_{2}\left(x_{t}+\lambda y_{t}\right)+\phi_{3} x_{t}+\phi_{4}-\frac{1}{\theta} \ln E_{t} e^{-\theta \phi_{2} \varepsilon_{t+1}}\right]^{\frac{\eta-1}{\eta}}$.

\footnotetext{
${ }^{19}$ Interest rates and income risk are important because they can be easily influenced by government policies. Economic growth may also be an important factor considering business cycles during which an economy typically moves from a trough to a peak over about five years.
} 
The F.O.C. and Envelope equation are thus

$$
\begin{aligned}
& \frac{\eta-1}{\eta}(1-p \beta) c_{t}^{-\frac{1}{\eta}}-\frac{\eta-1}{\eta} \beta p Q^{-\frac{1}{\eta}} \phi_{1}=0, \\
& \frac{\eta-1}{\eta} V^{-\frac{1}{\eta}}\left(A_{t-1}, x_{t-1}, y_{t}\right) \phi_{1}=\frac{\eta-1}{\eta} \beta p Q^{-\frac{1}{\eta}} \phi_{1} \hat{r},
\end{aligned}
$$

where

$$
Q \equiv \phi_{1}\left(\hat{r} A_{t-1}+y_{t}-c_{t}\right)+\phi_{2}\left(x_{t}+\lambda y_{t}\right)+\phi_{3} x_{t}+\phi_{4}-\frac{1}{\theta} \ln E_{t} e^{-\theta \phi_{2} \varepsilon_{t+1}} .
$$

(A.3) then implies

$$
\begin{aligned}
& c_{t}=\left(\phi_{1} \beta p\right)^{-\eta}(1-p \beta)^{\eta} Q, \\
& V\left(A_{t-1}, x_{t-1}, y_{t}\right)=(\hat{r} \beta p)^{-\eta} Q .
\end{aligned}
$$

Hence,

$$
V\left(A_{t-1}, x_{t-1}, y_{t}\right)=\left(\frac{\phi_{1}}{\hat{r}}\right)^{\eta}(1-p \beta)^{-\eta} c_{t}
$$

Also, (A.4b) gives

$\phi_{1}\left(\hat{r} A_{t-1}+y_{t}-c_{t}\right)+\phi_{2}\left(x_{t}+\lambda y_{t}\right)+\phi_{3} x_{t}+\phi_{4}-\frac{1}{\theta} \ln E_{t} e^{-\theta \phi_{2} \varepsilon_{t+1}}=(\hat{r} \beta p)^{\eta} V\left(A_{t-1}, x_{t-1}, y_{t}\right)$.

Using (A.5),

$$
\begin{aligned}
& \phi_{1} \hat{r} A_{t-1}+\left(\phi_{1}+\lambda \phi_{2}\right) y_{t}+\left(\phi_{2}+\phi_{3}\right) x_{t}+\phi_{4}-\frac{1}{\theta} \ln E_{t} e^{-\theta \phi_{2} \varepsilon_{t+1}} \\
= & {\left[(\hat{r} \beta p)^{\eta}+(1-p \beta)^{\eta} \phi_{1}^{1-\eta} \hat{r}^{\eta}\right] V\left(A_{t-1}, x_{t-1}, y_{t}\right) . }
\end{aligned}
$$

Using (A.2), (A.6) implies

$$
\begin{aligned}
& \phi_{1} \hat{r}=\phi_{1} \hat{r}^{\eta}\left[(\beta p)^{\eta}+(1-p \beta)^{\eta} \phi_{1}^{1-\eta}\right], \\
& \phi_{1}+\lambda \phi_{2}=\phi_{2} \hat{r}^{\eta}\left[(\beta p)^{\eta}+(1-p \beta)^{\eta} \phi_{1}^{1-\eta}\right], \\
& \phi_{2}+\phi_{3}=\phi_{3} \hat{r}^{\eta}\left[(\beta p)^{\eta}+(1-p \beta)^{\eta} \phi_{1}^{1-\eta}\right], \\
& \phi_{4}-\frac{1}{\theta} \ln E_{t} e^{-\theta \phi_{2} \varepsilon_{t+1}}=\phi_{4} \hat{r}^{\eta}\left[(\beta p)^{\eta}+(1-p \beta)^{\eta} \phi_{1}^{1-\eta}\right] .
\end{aligned}
$$

(A.7a) gives

$$
\hat{r}=\hat{r}^{\eta}\left[(\beta p)^{\eta}+(1-p \beta)^{\eta} \phi_{1}^{1-\eta}\right] .
$$

Then, (A.7b), (A.7c) and (A.7d) give

$$
\phi_{1}+\lambda \phi_{2}=\phi_{2} \hat{r}, \quad \phi_{2}+\phi_{3}=\phi_{3} \hat{r}, \quad \phi_{4}-\frac{1}{\theta} \ln E_{t} e^{-\theta \phi_{2} \varepsilon_{t+1}}=\phi_{4} \hat{r}
$$


(A.8) and (A.9) then imply

$$
\begin{aligned}
\phi_{1} & =(\hat{r}-\delta)^{\frac{1}{1-\eta}}\left(\hat{r}-\delta^{1 / \eta}\right)^{\frac{\eta}{\eta-1}} \\
\phi_{2} & =\frac{\phi_{1}}{\hat{r}-\lambda} \\
\phi_{3} & =\frac{\phi_{1}}{r(\hat{r}-\lambda)} \\
\phi_{4} & =-\frac{\phi_{1}}{r(\hat{r}-\lambda)} \varepsilon^{*}
\end{aligned}
$$

(A.5) implies

$$
\begin{aligned}
c_{t} & =\left(\frac{\hat{r}}{\phi_{1}}\right)^{\eta}(1-p \beta)^{\eta}\left(\phi_{1} A_{t-1}+\phi_{2} y_{t}+\phi_{3} x_{t}+\phi_{4}\right) \\
& =\hat{r}^{\eta} \phi_{1}^{1-\eta}(1-p \beta)^{\eta}\left(A_{t-1}+\frac{y_{t}}{\hat{r}-\lambda}+\frac{x_{t}-\varepsilon^{*}}{r(\hat{r}-\lambda)}\right)=(\hat{r}-\delta)\left(A_{t-1}+\frac{x_{t}+r y_{t}-\varepsilon^{*}}{r(\hat{r}-\lambda)}\right) .
\end{aligned}
$$

\section{A.2. Aggregate Wealth}

Substituting (2.11) and (2.13) into (2.12) gives

$$
\begin{aligned}
W^{*} & =(1-p) \sum_{t=1}^{\infty} p^{t-1}\left[\delta^{t} W^{*}+\frac{\delta-\lambda}{\hat{r}-\lambda} \sum_{i=1}^{t} \delta^{t-i} y_{i}-\frac{\hat{r}-\delta}{r(\hat{r}-\lambda)} \sum_{i=1}^{t} \delta^{t-i} x_{i}+\frac{\hat{r}-\delta}{r(\hat{r}-\lambda)} \frac{1-\delta^{t}}{1-\delta} \varepsilon^{*}\right] \\
& =\frac{1-p}{p}\left[\frac{p \delta}{1-p \delta} W+\frac{\delta-\lambda}{\hat{r}-\lambda} \sum_{t=1}^{\infty} \sum_{i=1}^{t} p^{t} \delta^{t-i} y_{i}-\frac{\hat{r}-\delta}{r(\hat{r}-\lambda)} \sum_{t=1}^{\infty} \sum_{i=1}^{t} p^{t} \delta^{t-i} x_{i}+\frac{\hat{r}-\delta}{r(\hat{r}-\lambda)} \varepsilon^{*} \sum_{t=1}^{\infty} p^{t} \frac{1-\delta^{t}}{1-\delta}\right]
\end{aligned}
$$

if $p \delta<1$. Then,

$$
\frac{p}{1-p}\left[1-\frac{(1-p) \delta}{1-p \delta}\right] W^{*}=\frac{\delta-\lambda}{\hat{r}-\lambda} \sum_{t=1}^{\infty} \sum_{i=1}^{t} p^{t} \delta^{t-i} y_{i}-\frac{\hat{r}-\delta}{r(\hat{r}-\lambda)} \sum_{t=1}^{\infty} \sum_{i=1}^{t} p^{t} \delta^{t-i} x_{i}+\frac{\hat{r}-\delta}{r(\hat{r}-\lambda)} \varepsilon^{*} \sum_{t=1}^{\infty} p^{t} \frac{1-\delta^{t}}{1-\delta}
$$

implying

$$
\frac{p}{1-p} \frac{1-\delta}{1-p \delta} W^{*}=\frac{\delta-\lambda}{\hat{r}-\lambda} \sum_{t=1}^{\infty} \sum_{i=1}^{t} p^{t} \delta^{t-i} y_{i}-\frac{\hat{r}-\delta}{r(\hat{r}-\lambda)} \sum_{t=1}^{\infty} \sum_{i=1}^{t} p^{t} \delta^{t-i} x_{i}+\frac{\hat{r}-\delta}{r(\hat{r}-\lambda)} \frac{p \varepsilon^{*}}{(1-p)(1-p \delta)} .
$$

We have

$$
\sum_{i=1}^{t} \delta^{t-i} x_{i}=\frac{\hat{a}-\lambda}{\hat{a}-\delta}\left[\hat{a}^{t}-\delta^{t}\right] \hat{a} y_{0}
$$

Thus, if $p \hat{a}<1$,

$$
\sum_{t=1}^{\infty} \sum_{i=1}^{t} p^{t} \delta^{t-i} x_{i}=\frac{\hat{a}-\lambda}{\hat{a}-\delta} \hat{a} y_{0} \sum_{t=1}^{\infty} p^{t}\left(\hat{a}^{t}-\delta^{t}\right)=\frac{p(\hat{a}-\lambda) \hat{a} y_{0}}{(1-p-a p)(1-p \delta)} .
$$


Substituting this into (A.10) then gives

$$
\frac{1-\delta}{1-p} W^{*}=\frac{1-p \delta}{p} \frac{\delta-\lambda}{\hat{r}-\lambda} \sum_{t=1}^{\infty} \sum_{i=1}^{t} p^{t} \delta^{t-i} y_{i}-\frac{(\hat{r}-\delta)(\hat{a}-\lambda) \hat{a} y_{0}}{r(\hat{r}-\lambda)(1-p-a p)}+\frac{\hat{r}-\delta}{r(\hat{r}-\lambda)} \frac{\varepsilon^{*}}{(1-p)}
$$

which immediately implies (2.14).

\section{A.3. The Steady State Case}

In the steady state, $y_{t}=x_{t}+\lambda y_{t-1}$, which can be easily solved:

$$
\begin{aligned}
y_{t} & =x_{t}+\lambda y_{t-1}=x_{t}+\lambda x_{t-1}+\lambda^{2} y_{t-2}+\cdots=\sum_{i=0}^{t-1} \lambda^{i} x_{t-i}+\lambda^{t} y_{0} \\
& =\hat{a}^{t} y_{0}\left[1-\left(\frac{\lambda}{\hat{a}}\right)^{t}\right]+\lambda^{t} y_{0}=\hat{a}^{t} y_{0} .
\end{aligned}
$$

Using this, we can write (2.14) in a simpler form. We have

$$
\sum_{i=1}^{t} \delta^{t-i} y_{i}=\delta^{t} y_{0} \sum_{i=1}^{t}\left(\frac{\hat{a}}{\delta}\right)^{i}=\frac{\hat{a}}{\delta-1-a}\left(\delta^{t}-\hat{a}^{t}\right) y_{0}
$$

and

$$
\sum_{t=1}^{\infty} \sum_{i=1}^{t} p^{t} \delta^{t-i} y_{i}=\frac{\hat{a} y_{0}}{\delta-1-a} \sum_{t=1}^{\infty} p^{t}\left(\delta^{t}-\hat{a}^{t}\right)=\frac{p \hat{a} y_{0}}{(1-p \delta)(1-p-a p)}
$$

Hence,

$$
W^{*}=\frac{1-p}{1-\delta} \frac{\delta-\lambda}{\hat{r}-\lambda} \frac{\hat{a}}{1-p-a p} y_{0}-\frac{(1-p)(\hat{r}-\delta)(\hat{a}-\lambda) \hat{a} y_{0}}{r(\hat{r}-\lambda)(1-\delta)(1-p-a p)}+\frac{\hat{r}-\delta}{r(\hat{r}-\lambda)(1-\delta)} \varepsilon^{*},
$$

which gives (2.16).

With the steady state economic growth in (2.15), the individual wealth profile in (2.11) becomes

$$
A_{t}=\delta^{t} A_{0}-\frac{r(\delta-\lambda)-(\hat{r}-\delta)(\hat{a}-\lambda)}{r(\hat{r}-\lambda)(\hat{a}-\delta)}\left(\delta^{t}-\hat{a}^{t}\right) \hat{a} y_{0}+\frac{\hat{r}-\delta}{r(\hat{r}-\lambda)} \frac{1-\delta^{t}}{1-\delta} \varepsilon^{*} .
$$

In equilibrium $A_{0}=W$, substituting (2.16) into (A.11) gives the equilibrium individual 
wealth profile:

$$
\begin{aligned}
A_{t}^{*}= & \delta^{t} W^{*}-\frac{r(\delta-\lambda)-(\hat{r}-\delta)(\hat{a}-\lambda)}{r(\hat{r}-\lambda)(\hat{a}-\delta)}\left(\delta^{t}-\hat{a}^{t}\right) \hat{a} y_{0}+\frac{\hat{r}-\delta}{r(\hat{r}-\lambda)} \frac{1-\delta^{t}}{1-\delta} \varepsilon^{*} \\
= & \frac{\delta^{t}(1-p) \hat{a} y_{0}}{r(1-\delta)(\hat{r}-\lambda)(1-p-a p)}[r(\delta-\lambda)-(\hat{r}-\delta)(\hat{a}-\lambda)]+\frac{(\hat{r}-\delta) \delta^{t}}{r(\hat{r}-\lambda)(1-\delta)} \varepsilon^{*} \\
& -\frac{r(\delta-\lambda)-(\hat{r}-\delta)(\hat{a}-\lambda)}{r(\hat{r}-\lambda)(\hat{a}-\delta)}\left(\delta^{t}-\hat{a}^{t}\right) \hat{a} y_{0}+\frac{\hat{r}-\delta}{r(\hat{r}-\lambda)} \frac{1-\delta^{t}}{1-\delta} \varepsilon^{*} \\
= & -\frac{r(\delta-\lambda)-(\hat{r}-\delta)(\hat{a}-\lambda)}{r(\hat{r}-\lambda)(1-p-a p)}\left\{\frac{1-p-a p}{\hat{a}-\delta}\left(\delta^{t}-\hat{a}^{t}\right)-\frac{1-p}{1-\delta} \delta^{t}\right\} \hat{a} y_{0}+\frac{\hat{r}-\delta}{r(\hat{r}-\lambda)} \frac{1}{1-\delta} \varepsilon^{*},
\end{aligned}
$$

which immediately implies (2.17). Substituting (2.17) into (2.10) then gives

$$
\begin{aligned}
c_{t}^{*}= & (\hat{r}-\delta)\left\{\frac{\hat{a}[r(\delta-\lambda)-(\hat{r}-\delta)(\hat{a}-\lambda)]}{r(\hat{r}-\lambda)(1-p-a p)(\hat{a}-\delta)}\left[\frac{1-\delta p}{1-\delta} a \delta^{t-1}+(1-p-a p) \hat{a}^{t-1}\right] y_{0}\right. \\
& \left.+\frac{\hat{r}-\delta}{r(\hat{r}-\lambda)} \frac{1}{1-\delta} \varepsilon^{*}+\frac{x_{t}+r y_{t}-\varepsilon^{*}}{r(\hat{r}-\lambda)}\right\} \\
= & \frac{(\hat{r}-\delta) \hat{a}[r(\delta-\lambda)-(\hat{r}-\delta)(\hat{a}-\lambda)]}{r(\hat{r}-\lambda)(1-p-a p)(\hat{a}-\delta)}\left[\frac{1-\delta p}{1-\delta} a \delta^{t-1}+(1-p-a p) \hat{a}^{t-1}\right] y_{0} \\
& +\frac{\hat{r}-\delta}{r(\hat{r}-\lambda)}\left(x_{t}+r y_{t}\right)+\frac{\hat{r}-\delta}{(\hat{r}-\lambda)(1-\delta)} \varepsilon^{*} .
\end{aligned}
$$

That is,

$$
\begin{aligned}
c_{t}^{*}= & \frac{\hat{r}-\delta}{\hat{r}-\lambda}\left\{\frac{r(\delta-\lambda)-(\hat{r}-\delta)(\hat{a}-\lambda)}{r(1-p-a p)(\hat{a}-\delta)}\left[\frac{1-\delta p}{1-\delta} a \delta^{t-1}+(1-p-a p) \hat{a}^{t-1}\right] \hat{a} y_{0}\right. \\
& \left.+\frac{x_{t}}{r}+y_{t}+\frac{1}{1-\delta} \varepsilon^{*}\right\},
\end{aligned}
$$

which immediately gives (2.18).

\section{References}

[1] Caballero, Ricardo (1990): "Consumption Puzzles and Precautionary Savings", Journal of Monetary Economics, 25, 113-136.

[2] Caballero, Ricardo (1991): "Earnings Uncertainty and Aggregate Wealth Accumulation", American Economic Review, 81, 859-872.

[3] Carroll, Christopher D. (1992): "The Buffer-Stock Theory of Saving: Some Macroeconomics Evidence", Brookings Papers on Economic Activity, No.2, 61-135.

[4] Carroll, Christopher D. and David N. Weil (1993): "Saving and Growth: A Reinterpretation", NBER Working Paper, No.4470. 
[5] Epstein, Larry G. and Stanley E. Zin (1989): "Substitution, Risk Aversion and the Temporal Behavior of Consumption and Asset Returns: A Theoretical Framework", Econometrica, 57, 937-969.

[6] Wood, J. Cunningham and Ronald N. Woods, eds (1990): Milton Friedman: Critical assessments, London and New York: Routledge.

[7] Irvine, Ian and Susheng Wang (1994): "Earnings Uncertainty and Wealth Accumulation: Comment", American Economic Review, 84, 1463-1469.

[8] Kotlikoff, Laurence J. (1988): "Intergenerational Transfers and Savings", Journal of Economic Perspectives, 2, 41-58.

[9] Kreps, David M. and Evan L. Porteus (1978): "Temporal Resolution of Uncertainty and Dynamic Choice Theory", Econometrica, 46, 185-200.

[10] McCallum, Bennett T. (1989): Monetary Economics: Theory and Policy, Macmillan Publishing Company.

[11] Modigliani, Franco and R. Brumberg (1954): "Utility Analysis and the Consumption Function: An Interpretation of Cross Section Data", in K. Kurihara ed., Post-Keynesian Economics, New Brunswick, NJ: Rutgers University Press, 338436.

[12] Prescott, Edward C. (1986): "Theory Ahead of Business Cycle Measurement," Carnegie-Rochester Conference Series on Public Policy, 25, 11-44, Autumn.

[13] Skinner, Jonathan (1988): "Risky Income, Life Cycle Consumption, and Precautionary Savings", Journal of Monetary Economics, 22, 237-255.

[14] Summers, Lawrence H. (1981): "Capital Taxation and Accumulation in a Life Cycle Growth Model," American Economic Review, 74, 533-545.

[15] Wang, Susheng (1998): "Saving and Wealth in an Intergenerational Equilibrium", Journal of Monetary Economics, revision and resubmission.

[16] Weil, Philippe (1993): "Precautionary Savings and the Permanent Income Hypothesis", Review of Economic Studies, 60, 367-384.

[17] Yaari, Menahem (1964): "On the Consumer's Lifetime Allocation Process", International Economic Review, 5, 304-317.

[18] Zeldes, Stephen (1989): "Optimal Consumption with Stochastic Income", Quarterly Journal of Economics, 104, 275-298. 